\title{
ON THE HAZARD FUNCTION OF BirnBAUM-SAUNDERS Distribution AND Associated INFERENCE
}

\author{
Debasis KUndu* ${ }^{*} \quad$ NANDini KAnNAN ${ }^{\dagger}$ \& N. BALAKRISHNAN ${ }^{\ddagger}$
}

\begin{abstract}
In this paper, we discuss the shape of the hazard function of Birnbaum-Saunders distribution. Specifically, we establish that the hazard function of Birnbaum-Saunders distribution is an upside down function for all values of the shape parameter. In reliability and survival analysis, as it is often of interest to determine the point at which the hazard function reaches its maximum, we propose different estimators of that point and evaluate their performance using Monte Carlo simulations. Next, we analyze a data set and illustrate all the inferential methods developed here and finally make some concluding remarks.
\end{abstract}

Keywords and Phrases: Birnbaum-Saunders distribution; hazard function; change point; asymptotic distribution; Bootstrap confidence interval; Modified moment estimators; Biascorrected modified moment estimators; Maximum likelihood estimators.

Short Running Title: Hazard Analysis for Birnbaum-Saunders Distribution

*Department of Mathematics and Statistics, Indian Institute of Technology Kanpur, Pin 208016, INDIA. Corresponding Author

${ }^{\dagger}$ Department of Management Science and Statistics, The University of Texas at San Antonio, 1604 N.West Loop, San Antonio, Texas 78249, USA.

${ }^{\ddagger}$ Department of Mathematics and Statistics, McMaster University, Hamilton, Ontario, Canada L8S 4K1 


\section{INTRODUCTION}

The two-parameter Birnbaum-Saunders (BS) distribution was originally proposed by Birnbaum and Saunders $[4,5]$ as a failure time distribution for fatigue failure caused under cyclic loading. The cumulative distribution function (CDF) of a two-parameter BS random variable $T$ is of the form

$$
F(t ; \alpha, \beta)=\Phi\left[\frac{1}{\alpha}\left\{\left(\frac{t}{\beta}\right)^{\frac{1}{2}}-\left(\frac{\beta}{t}\right)^{\frac{1}{2}}\right\}\right], \quad 0<t<\infty, \quad \alpha, \beta>0,
$$

where $\Phi(\cdot)$ is the standard normal CDF. The parameters $\alpha$ and $\beta$ in (1) are the shape and scale parameters, respectively. Although the BS distribution was originally proposed as a failure time distribution for fatigue failure under the assumption that the failure is due to development and growth of a dominant crack, a more general derivation was provided by Desmond [9] based on a biological model. Desmond [9] also strengthened the physical justification for the use of this distribution by relaxing the assumptions made originally by Birnbaum and Saunders [4]. Some recent work on the BS distribution can be found in Balakrishnan et al. [2], Chang and Tang [7, 8], Dupuis and Mills [10], From and Li [11], Lemonte et al. [16], Rieck [23, 24], Ng et al. [18, 19], Owen [20] and Xie and Wei [26]. A concise review of different developments on the BS distribution until 1995 can be found in the book by Johnson et al. [14].

It is known (see, for example, Johnson et al. [14]) that the density function of the BS distribution is unimodal. Though several articles have been published in the last three decades regarding different inferential methods for the parameters of the BS distribution and their properties, yet the shape of the hazard function has not been examined possibly due to its complex form. Mann et al. [17] mentioned that the hazard function of the BS

distribution is not an increasing function of $t$, although they did not provide a formal proof for it. In this paper, we first formally prove that the hazard function of the BS distribution 
is indeed an upside down function of $t>0$ for all values of the shape parameter $\alpha$ and scale parameter $\beta$.

It is not uncommon to model survival and failure time data by distributions which have monotone hazard function. But in many practical situations, the hazard function is not monotone and in fact it increases up to a point and then decreases. For example, in the study of recovery from breast cancer, it has been observed by Langlands et al. [15] that the maximum mortality occurs after about three years and then it decreases slowly over a fixed period of time. Yet another example concerns the Veteran Administration lung cancer trial which revealed that the failure rates for both low and high performance status groups are upside down (Bennett [3]). In these cases, a quantity of natural interest is the point at which the hazard function is maximum; see Gupta et al. [13]. Hereafter, we refer to this point as the change point of the hazard function as the monotonicity changes from increasing to decreasing at that point.

In this paper, we investigate the change point of the hazard function of the BS distribution and discuss some methods of estimation for that change point.

Since the change point is a function of the two parameters of the BS distribution, it would be logical to estimate the change point by replacing the parameters by their estimates. Several estimation methods have been discussed by $\mathrm{Ng}$ et al. [18] who have observed that the modified moment estimators (MMEs) and the bias-corrected modified moment estimators (BCMMEs), in particular, are quite efficient and are also easy to implement computationally. For this reason, we propose to estimate the change point by replacing the two unknown parameters by the corresponding MMEs and BCMMEs. Next, we obtain the asymptotic distributions of these estimators of the change point. We also suggest the use of nonparametric bootstrap method to obtain confidence intervals for the change point. We then evaluate the performance of these estimators in terms of bias and variance by means of 
Monte Carlo simulations for small, moderate and large sample sizes. Finally, we analyze a real data set and illustrate all the inferential methods discussed here.

In Section 2, we briefly describe some basic characteristics of the BS distribution. In Section 3, we establish formally that the shape of the hazard function of the BS distribution is upside down. In Section 4, we show that the change point of the hazard function can be determined as a solution of a non-linear equation. An approximation of this change point is also suggested. In Section 5, different methods of estimation of the change point are discussed. In Section 6, some comparative results based on Monte Carlo simulations are presented. After a data set is analyzed in Section 7, some concluding remarks are finally made in Section 8.

\section{Birnbaum-Saunders Distribution}

The probability density function (PDF) of a two-parameter BS random variable $T$ corresponding to the CDF in (1) is given by

$f(t ; \alpha, \beta)=\frac{1}{2 \sqrt{2 \pi} \alpha \beta}\left[\left(\frac{\beta}{t}\right)^{1 / 2}+\left(\frac{\beta}{t}\right)^{3 / 2}\right] \exp \left[-\frac{1}{2 \alpha^{2}}\left(\frac{t}{\beta}+\frac{\beta}{t}-2\right)\right], 0<t<\infty, \alpha, \beta>0$.

Consider now the monotone transformation

$$
X=\frac{1}{2}\left[\left(\frac{T}{\beta}\right)^{\frac{1}{2}}-\left(\frac{T}{\beta}\right)^{-\frac{1}{2}}\right]
$$

or

$$
T=\beta\left\{1+2 X^{2}+2 X\left(1+X^{2}\right)^{\frac{1}{2}}\right\}
$$

then from (1), it readily follows that $X$ is distributed as normal with mean zero and variance $\left(\alpha^{2} / 4\right)$. The transformation in (4) is a very useful transformation as it enables the determination of the moments of $T$ through known results on expectations of functions of $X$. For 
example, we have:

$$
\begin{gathered}
E(T)=\beta\left(1+\frac{1}{2} \alpha^{2}\right), \\
V(T)=(\alpha \beta)^{2}\left(1+\frac{5}{4} \alpha^{2}\right), \\
\beta_{1}(T)=\frac{16 \alpha^{2}\left(11 \alpha^{2}+6\right)}{\left(5 \alpha^{2}+4\right)^{3}}, \\
\beta_{2}(T)=3+\frac{6 \alpha^{2}\left(93 \alpha^{2}+41\right)}{\left(5 \alpha^{2}+4\right)^{2}},
\end{gathered}
$$

where $E(T), V(T), \beta_{1}(T)$ and $\beta_{2}(T)$ are the expected value, variance, coefficient of skewness, and coefficient of kurtosis, respectively. It can also be easily shown that if $T$ has a BS distribution with parameters $\alpha$ and $\beta$, then $T^{-1}$ also has a BS distribution with parameters $\alpha$ and $\beta^{-1}$, respectively.

\section{SHAPE OF THE HAZARD}

To examine the shape of the hazard function, let us assume that the scale parameter $\beta=1$, without loss of any generality. Let us consider the function

$$
\epsilon(t)=t^{\frac{1}{2}}-t^{-\frac{1}{2}}
$$

for which

$$
\epsilon^{\prime}(t)=\frac{d}{d t} \epsilon(t)=\frac{1}{2}\left(t^{-\frac{1}{2}}+t^{-\frac{3}{2}}\right)=\frac{1}{2 t}\left(t^{\frac{1}{2}}+t^{-\frac{1}{2}}\right), \quad \epsilon^{\prime \prime}(t)=\frac{d}{d t} \epsilon^{\prime}(t)=-\frac{1}{4 t^{2}}\left(t^{\frac{1}{2}}+3 t^{-\frac{1}{2}}\right),
$$

and also

$$
\epsilon^{2}(t)=t+\frac{1}{t}-2
$$

The density function of the BS distribution in $(2)$ (for $\beta=1$ ) is then

$$
f(t ; \alpha)=\frac{1}{\sqrt{2 \pi} \alpha} \epsilon^{\prime}(t) e^{-\frac{1}{2 \alpha^{2}} \epsilon^{2}(t)}
$$


which, in conjunction with the expression of the distribution function in (1), gives the hazard function as

$$
h(t ; \alpha)=\frac{f(t ; \alpha)}{1-F(t ; \alpha, 1)}=\frac{\frac{1}{\sqrt{2 \pi} \alpha} \epsilon^{\prime}(t) e^{-\frac{1}{2 \alpha^{2}} \epsilon^{2}(t)}}{\Phi\left(-\frac{\epsilon(t)}{\alpha}\right)} .
$$

From (13), the shape of $h(t ; \alpha)$ is not at all clear. We need the following lemmas for establishing our main result regarding the shape of the hazard function $h(t ; \alpha)$ in $(13)$.

Lemma 1: Suppose $f(t)$, for $t>0$, is the density function of a positive real-valued continuous random variable, $f^{\prime}(t)$ is the derivative of $f(t)$, and $\eta(t)=-\frac{f^{\prime}(t)}{f(t)}$. Then, if there exists a $t_{0}$ such that $\eta^{\prime}(t)>0 \forall t \in\left(0, t_{0}\right), \eta^{\prime}\left(t_{0}\right)=0$ and $\eta^{\prime}(t)<0 \forall t \in\left(t_{0}, \infty\right)$, the hazard function corresponding to $f(t)$ is either an upside down or a decreasing function of $t$.

PRoOF: See Glaser [12].

Lemma 2: The hazard function of Birnbaum-Saunders distribution is either an upside down or a decreasing function of $t>0$, for all values of the shape parameter $\alpha$.

Proof: In this case, upon differentiating $f(t ; \alpha)$ in $(12)$ with respect to $t$, we readily get

$$
f^{\prime}(t ; \alpha)=\frac{1}{\sqrt{2 \pi} \alpha} e^{-\frac{1}{2 \alpha^{2}} \epsilon^{2}(t)}\left(\epsilon^{\prime \prime}(t)-\frac{1}{\alpha^{2}}\left(\epsilon^{\prime}(t)\right)^{2} \epsilon(t)\right)
$$

consequently, we obtain

$$
\eta(t ; \alpha)=-\frac{f^{\prime}(t ; \alpha)}{f(t ; \alpha)}=-\left[\frac{\epsilon^{\prime \prime}(t)}{\epsilon^{\prime}(t)}-\frac{\epsilon(t) \epsilon^{\prime}(t)}{\alpha^{2}}\right]=\frac{1}{2 t}+\frac{1}{t(t+1)}+\frac{1}{2 \alpha^{2}}-\frac{1}{2 \alpha^{2} t^{2}}
$$

and

$$
\eta^{\prime}(t ; \alpha)=\frac{s(t ; \alpha)}{2(t+1)^{2} \alpha^{2} t^{3}},
$$

where

$$
s(t ; \alpha)=-\alpha^{2} t^{3}+\left(-6 \alpha^{2}+2\right) t^{2}+\left(-3 \alpha^{2}+4\right) t+2 .
$$


Observe that $s(0 ; \alpha)=2$ and $s(\infty ; \alpha)=\lim _{t \rightarrow \infty} s(t)=-\infty$. It is clear that the signs of $s(t ; \alpha)$ and $\eta^{\prime}(t ; \alpha)$ are the same $\forall t>0$. Let us consider the roots of

$$
s^{\prime}(t ; \alpha)=-3 \alpha^{2} t^{2}+2\left(-6 \alpha^{2}+2\right) t+\left(-3 \alpha^{2}+4\right)=0 .
$$

Since the discriminant of the above quadratic equation

$$
4\left(27 \alpha^{4}-12 \alpha^{2}+4\right)=4\left\{\left(3 \alpha^{2}-2\right)^{2}+18 \alpha^{4}\right\}
$$

is always positive, both roots of the quadratic equation in (14) are real and are given by

$$
\frac{-2\left(-6 \alpha^{2}+2\right) \pm \sqrt{4\left(-6 \alpha^{2}+2\right)^{2}+12 \alpha^{2}\left(-3 \alpha^{2}+4\right)}}{-6 \alpha^{2}} .
$$

It can be easily seen that if $0<\alpha<\sqrt{\frac{4}{3}}$, one root is positive and the other one is negative. Let us denote the two roots by $r_{1}$ and $r_{2}$, where $r_{1}<r_{2}$.

CASE 1: $0<\alpha<\sqrt{\frac{4}{3}}$

In this case, $r_{1}<0, r_{2}>0$ and $s^{\prime}(0 ; \alpha)=-3 \alpha^{2}+4>0$. Hence, $s^{\prime}(t ; \alpha)<0$ for $t<r_{1}$, $s^{\prime}(t ; \alpha)>0$ for $r_{1}<t<r_{2}$, and $s^{\prime}(t ; \alpha)<0$ for $t>r_{2}$. This implies that $s(t ; \alpha)$ is a decreasing function of $t$ from $-\infty$ to $r_{1}$, is an increasing function of $t$ from $r_{1}$ to $r_{2}$, and is a decreasing function of $t$ again from $r_{2}$ to $\infty$. Now, upon using the facts that $s(0 ; \alpha)=2$ and $s(\infty ; \alpha)=-\infty$, it readily follows that there exists a $t_{0}$ such that $s(t ; \alpha)>0 \forall t \in\left(0, t_{0}\right)$, $s\left(t_{0} ; \alpha\right)=0$, and $s(t ; \alpha)<0 \forall t>t_{0}$.

CASE 2: $\alpha \geq \sqrt{\frac{4}{3}}$

In this case, by writing the second term of $s^{\prime}(t ; \alpha)$ in $(14)$ as $2\left(-6 \alpha^{2}+8\right) t-12 t$, we see that $s^{\prime}(t ; \alpha)<0$ for all $t>0$. Hence, $s(t ; \alpha)$ is a decreasing function of $t$ for $t>0$ and it decreases from 2 to $-\infty$ which readily implies that there exists a $t_{0}>0$ such that $s(t ; \alpha)>0 \forall t \in$ $\left(0, t_{0}\right), s\left(t_{0} ; \alpha\right)=0$, and $s(t ; \alpha)<0 \forall t>t_{0}$. 
The proof of this lemma is then completed upon using Lemma 1.

LEMMA 3: For $\alpha>0$, the hazard function of the BS distribution is indeed an upside down function.

Proof: Note that it is enough to prove that $\lim _{t \rightarrow 0} h(t ; \alpha)=0$. From (13), we have

$$
h(t ; \alpha)=\frac{\frac{\epsilon^{\prime}(t)}{\alpha} \phi\left(\frac{\epsilon(t)}{\alpha}\right)}{\Phi\left(-\frac{\epsilon(t)}{\alpha}\right)} .
$$

Since $\lim _{t \rightarrow 0} \Phi\left(-\frac{\epsilon(t)}{\alpha}\right)=1$, we consider only the numerator of $h(t ; \alpha)$. Note that

$$
\frac{1}{\alpha} \epsilon^{\prime}(t) \phi\left(\frac{\epsilon(t)}{\alpha}\right)=k e^{-\frac{(\epsilon(t))^{2}}{2 \alpha^{2}}}\left(t^{-\frac{1}{2}}+t^{-\frac{3}{2}}\right),
$$

where $k$ is a positive constant. Now consider

$$
\lim _{t \rightarrow 0}\left[\ln \left(e^{-\frac{(\epsilon(t))^{2}}{2 \alpha^{2}}} t^{-\frac{1}{2}}\right)\right]=\lim _{t \rightarrow 0}\left[-\frac{(\epsilon(t))^{2}}{2 \alpha^{2}}-\frac{1}{2} \ln t\right]=\lim _{t \rightarrow 0} \frac{1}{2 \alpha^{2}}\left[-t-t^{-1}+2-\alpha^{2} \ln t\right]=-\infty .
$$

Therefore,

$$
\lim _{t \rightarrow 0}\left[e^{-\frac{(\epsilon(t))^{2}}{2 \alpha^{2}}} t^{-\frac{1}{2}}\right]=0
$$

Similarly, it can be shown that

$$
\lim _{t \rightarrow 0}\left[e^{-\frac{(\epsilon(t))^{2}}{2 \alpha^{2}}} t^{-\frac{3}{2}}\right]=0
$$

which completes the proof of the lemma.

By combining all these results, we can now state the following result.

ThEOREM 1: The hazard function of the BS distribution is an upside down function for all values of the shape parameter $\alpha$. 


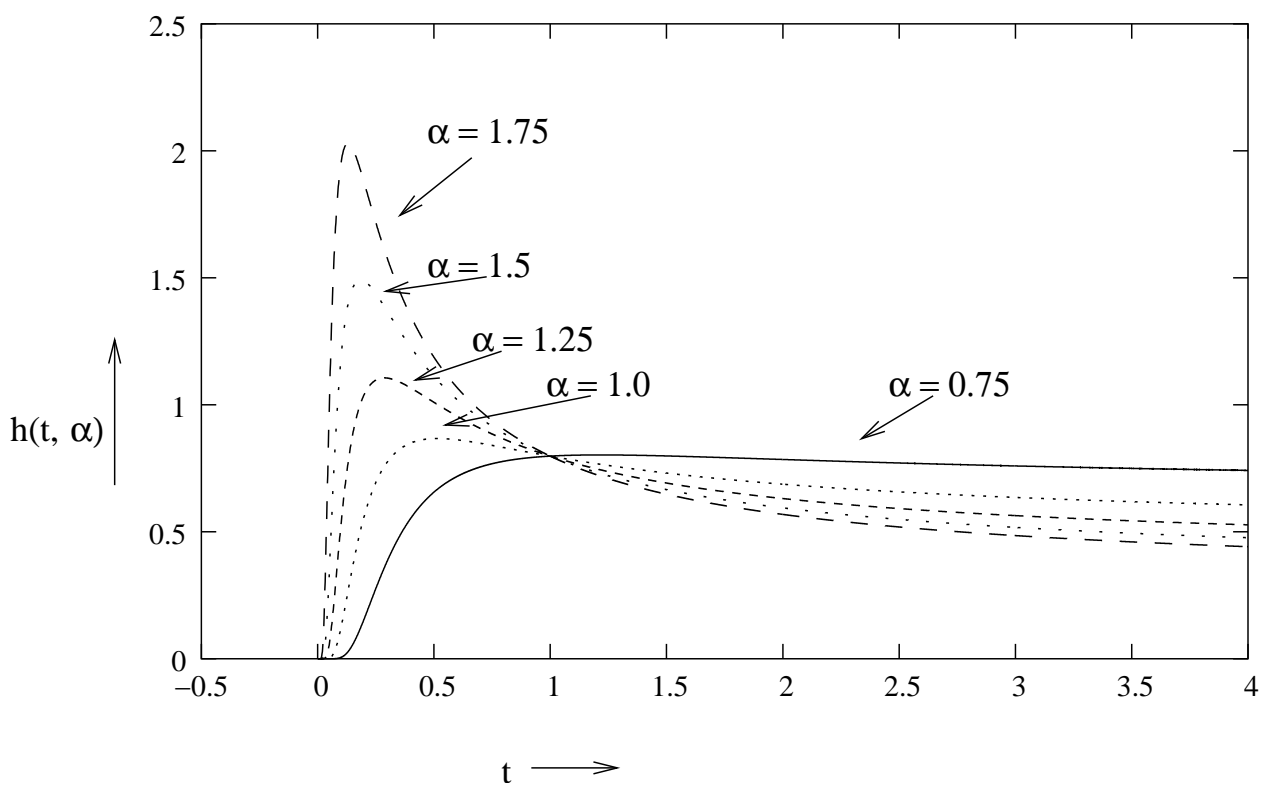

Figure 1: Hazard functions of the Birnbaum-Saunders distribution for different values of $\alpha$, when $\beta=1$.

\section{Change Point of the Hazard}

In the preceding section, we established that the hazard function of the BS distribution is an upside down function. It is then of natural interest to study the change point of the hazard function.

Since $\beta$ is the scale parameter, it is evident that if $c_{\alpha, \beta}$ is the change point of $h(t ; \alpha, \beta)$, then

$$
c_{\alpha, \beta}=\beta c_{\alpha, 1} .
$$

For this reason, we assume $\beta=1$ (without loss of any generality) for the remainder of this section, and for notational simplicity we denote $c_{\alpha, 1}$ by $c_{\alpha}$. In Figure 1, we have presented plots of the hazard function of the BS distribution for different values of $\alpha$. Note that the change point $c_{\alpha}$ is a decreasing function of $\alpha$, which can be obtained as the solution of the 
Table 1: Values of $c_{\alpha}$ for different choices of $\alpha$

\begin{tabular}{|l|l||l|l||l|l||l|l||l|l||}
\hline$\alpha$ & $c_{\alpha}$ & $\alpha$ & $c_{\alpha}$ & $\alpha$ & $c_{\alpha}$ & $\alpha$ & $c_{\alpha}$ & $\alpha$ & $c_{\alpha}$ \\
\hline 0.6 & 2.5364 & 1.0 & 0.5149 & 1.4 & 0.2178 & 1.8 & 0.1221 & 2.2 & 0.0787 \\
0.7 & 1.5233 & 1.1 & 0.3981 & 1.5 & 0.1850 & 1.9 & 0.1083 & 2.3 & 0.0716 \\
0.8 & 0.9923 & 1.2 & 0.3180 & 1.6 & 0.1594 & 2.0 & 0.0968 & 2.4 & 0.0654 \\
0.9 & 0.6950 & 1.3 & 0.2605 & 1.7 & 0.1388 & 2.1 & 0.0870 & 2.5 & 0.0599 \\
\hline
\end{tabular}

following non-linear equation;

$$
\Phi\left(-\frac{1}{\alpha} \epsilon(t)\right)\left\{-\left(\epsilon^{\prime}(t)\right)^{2} \epsilon(t)+\alpha^{2} \epsilon^{\prime \prime}(t)\right\}+\alpha \phi\left(-\frac{1}{\alpha} \epsilon(t)\right)\left(\epsilon^{\prime}(t)\right)^{2}=0 .
$$

There is no explicit solution for Eq. (15), and so it needs to be determined by numerical methods. In Table 1, we have presented the values of $c_{\alpha}$ for different choices of $\alpha$. It should be mentioned that the calculation of $c_{\alpha}$ from (15) is not difficult for large values of $\alpha$. Standard root solving technique like Newton-Raphson method works very well. But for smaller values of $\alpha$, finding $c_{\alpha}$ is extremely difficult. As $\alpha$ approaches zero, $c_{\alpha}$ approaches infinity. Particularly, it is observed that for $\alpha<0.5$, the numerical solution of (15) is very unstable, and so extreme care is needed to calculate $c_{\alpha}$ in this case. The standard root solving methods do not work. The problem can be avoided by using Mills' ratio available in the literature; see, for example, Pinelis [21]. It is not attempted here, as $\alpha$ approaches zero, the shape of the PDF of the Birnbaum-Saunders distribution becomes symmetric and degenerate at the scale parameter, and one may prefer to use some other symmetric distribution rather than a Birnbaum-Saunders distribution. Since Birnbaum-Saunders distribution is usually used to model skewed data, it will not be common in practice to have $\alpha$ close to zero.

Since $c_{\alpha}$ does not have a closed-form expression, we seek to obtain a functional approximation of $c_{\alpha}$ as a function of $\alpha$. Using Box-Cox type transformation, it was observed that $c_{\alpha}^{-\frac{1}{2}}$ is approximately a linear function of $\alpha$ and so a reasonably good approximation of $c_{\alpha}$, 


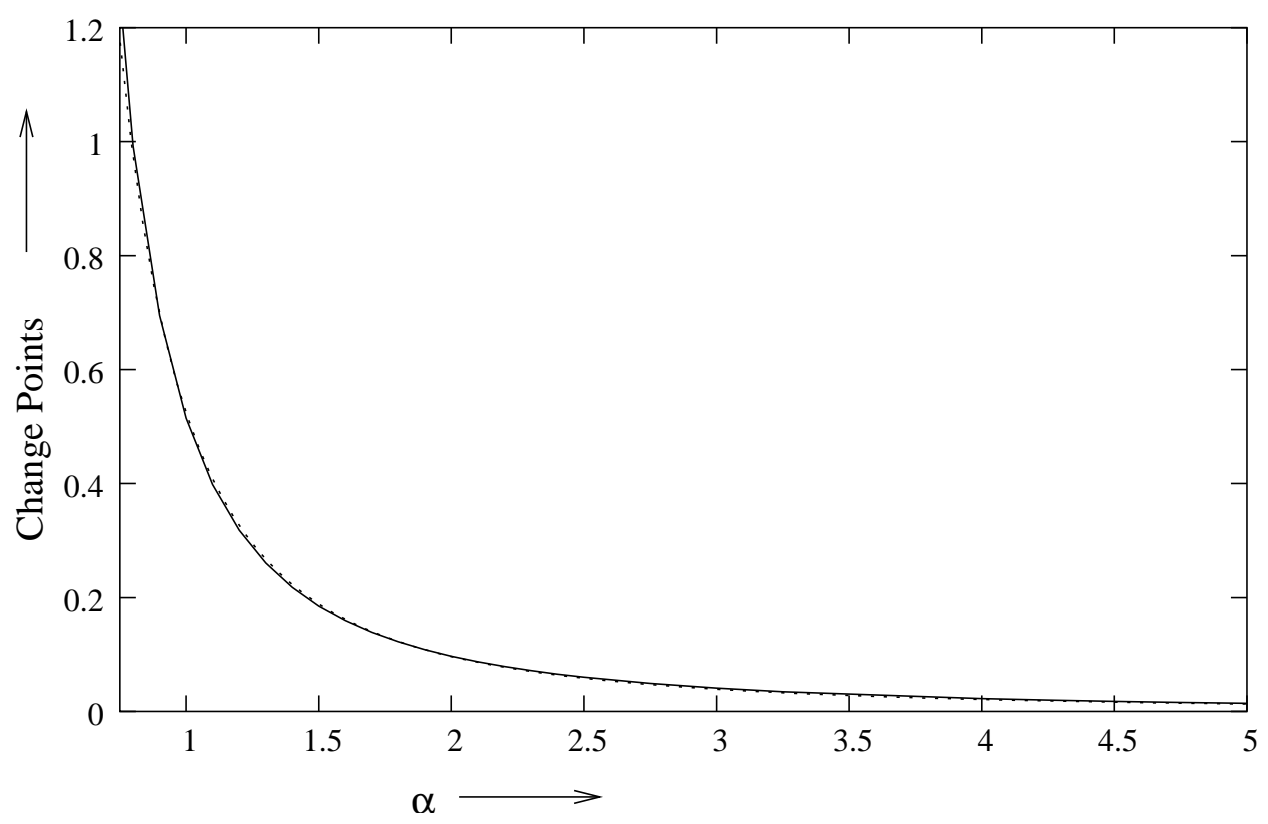

Figure 2: The change points and the approximate change points of the hazard functions of the Birnbaum-Saunders distribution for different values of $\alpha$, when $\beta=1$.

say $\tilde{c}_{\alpha}$, as a function of $\alpha$ is given by

$$
\tilde{c}_{\alpha}=\frac{1}{(-0.4604+1.8417 \alpha)^{2}}
$$

for $\alpha>\frac{0.4604}{1.8417}=0.25$. It is observed that for $\alpha>0.6$, the approximation in (16) works very well. Since the computation of $c_{\alpha}$ itself is very difficult for $0.25<\alpha<0.5$, it makes it difficult to find a reasonable approximation of $c_{\alpha}$ in that range. Plots of $c_{\alpha}$ and $\tilde{c}_{\alpha}$ against $\alpha$ are presented in Figure 2 where from it is observed that the approximation in (16) is quite accurate whenever $\alpha$ is not very small. 


\section{Estimation of the Change Point}

In this section, we propose different estimators for the change point $c_{\alpha, \beta}$ of the hazard function $h(t, \alpha, \beta)$ of the BS distribution, where

$$
h(t, \alpha, \beta)==\frac{f(t ; \alpha, \beta)}{1-F(t ; \alpha, \beta)}=\frac{\frac{1}{\sqrt{2 \pi} \alpha} \epsilon^{\prime}\left(\frac{t}{\beta}\right) e^{-\frac{1}{2 \alpha^{2}} \epsilon^{2}\left(\frac{t}{\beta}\right)}}{\Phi\left(-\frac{\epsilon\left(\frac{t}{\beta}\right)}{\alpha}\right)} .
$$

For this purpose, we use the modified moment estimators (MMEs) and the bias-corrected modified moment estimators (BCMMEs) of $\alpha$ and $\beta$ discussed by $\mathrm{Ng}$ et al. [18]. These authors observed that the MMEs are very simple to use and that they behave very much like maximum likelihood estimators (MLEs). They are also slightly biased, like the MLEs, in the case of small sample sizes and for this reason the bias-corrected estimators were proposed. The BCMMEs were shown to be good estimators in terms of both bias and variance. More importantly, as aptly mentioned by $\mathrm{Ng}$ et al. [18], both these estimators are very simple to implement as they do not require any non-linear root solving procedure as needed by the MLEs.

Let $t_{1}, \cdots, t_{n}$ be a random sample of size $n$ from the BS distribution with PDF as in (2). Let

$$
s=\frac{1}{n} \sum_{i=1}^{n} t_{i} \quad \text { and } \quad r=\left[\frac{1}{n} \sum_{t=1}^{n} \frac{1}{t_{i}}\right]^{-1}
$$

denote the sample arithmetic and harmonic means, respectively. Then, the MMEs of $\alpha$ and $\beta$ are given by

$$
\hat{\alpha}=\left(2\left[\left(\frac{s}{r}\right)^{\frac{1}{2}}-1\right]\right)^{\frac{1}{2}} \quad \text { and } \quad \hat{\beta}=(s r)^{\frac{1}{2}},
$$

and the BCMMEs are given by

$$
\tilde{\alpha}=\left(\frac{n}{n-1}\right) \hat{\alpha} \quad \text { and } \quad \tilde{\beta}=\left(1+\frac{\tilde{\alpha}^{2}}{4 n}\right)^{-1} \hat{\beta} ;
$$

see $\mathrm{Ng}$, Kundu and Balakrishnan [18]. We, therefore, propose to estimate $c_{\alpha, \beta}$ by $c_{\hat{\alpha}, \hat{\beta}}$ or $c_{\tilde{\alpha}, \tilde{\beta}}$. For simplicity, we shall call $c_{\hat{\alpha}, \hat{\beta}}$ and $c_{\tilde{\alpha}, \tilde{\beta}}$ as the MME and BCMME of $c_{\alpha, \beta}$, respectively. In 
a similar vein, $\tilde{c}_{\alpha}$ in (16) can be used in conjunction with MMEs and BCMMEs of $\alpha$ and $\beta$ to produce approximate MME (AMME) and approximate BCMME (ABCMME) of $c_{\alpha, \beta}$

as $\hat{\beta} \tilde{c}_{\hat{\alpha}}$ and $\tilde{\beta} \tilde{c}_{\tilde{\alpha}}$, respectively. The asymptotic distributions of all these estimators can be derived by using delta method, and the details are presented in the Appendix.

\section{Simulation Results}

We carried out Monte Carlo simulations in order to compare the performance of all the estimators proposed in the preceding section. We used several different sample sizes and parameter values. All the computations were performed using RAN2 random deviate generator of Press et al. [22], and the programs were written in FORTRAN-77.

The sample sizes considered were $n=10,15,25,50$ and the shape parameter was taken as $\alpha=0.75,1.00,1.50$ and 2.00 . In all cases, we set the scale parameter $\beta=1$. We used 1,000 replications to estimate the change point $c_{\alpha}$ by using the MME, BCMME, AMME and ABCMME methods. The values of the average and variance of all estimates were computed and these are reported in Table 2, wherein the the true value of $c_{\alpha}$ [determined numerically from (15)] are also presented for comparison purposes.

From the values in Table 2, it is clear that both bias and variance of all estimators decrease when the sample size increases, as one would expect. When the sample size is very small, say $n=10$, then none of the methods work satisfactorily. While all the methods overestimate $c_{\alpha}$ generally, the BCMME and ABCMME seem to possess smaller bias and variance than the MME and AMME, respectively. Since the performance of the estimators BCMME and ABCMME are quite similar and that they can be computed explicitly without requiring the use of any non-linear equation solver, we recommend the use of ABCMME for estimating the change point of the hazard function of the BS distribution as it shows a 
Table 2: Values of average and variance of different estimators of $c_{\alpha}$

\begin{tabular}{|c|c|c|c|c|c|c|}
\hline $\mathrm{n}$ & $\alpha$ & $c_{\alpha}$ & MME & BCMME & AMME & ABCMME \\
\hline 10 & 0.75 & 1.2162 & $1.5980(0.7204)$ & $1.3381(0.6534)$ & $1.8891(0.8148)$ & $1.4141(0.6570)$ \\
& 1.00 & 0.5149 & $0.8315(0.2475)$ & $0.6000(0.1486)$ & $0.8385(0.2041)$ & $0.6082(0.1021)$ \\
& 1.50 & 0.1850 & $0.3097(0.0559)$ & $0.2273(0.0275)$ & $0.3148(0.0434)$ & $0.2303(0.0233)$ \\
& 2.00 & 0.0968 & $0.1551(0.0091)$ & $0.1515(0.0180)$ & $0.1595(0.0112)$ & $0.1345(0.0204)$ \\
\hline 15 & 0.75 & 1.2162 & $1.5284(0.5704)$ & $1.2801(0.3953)$ & $1.6194(0.5043)$ & $1.2898(0.2997)$ \\
& 1.00 & 0.5149 & $0.7615(0.1959)$ & $0.5920(0.1207)$ & $0.7230(0.1287)$ & $0.5873(0.0823)$ \\
& 1.50 & 0.1850 & $0.2679(0.0333)$ & $0.2154(0.0190)$ & $0.2703(0.0290)$ & $0.2068(0.0099)$ \\
& 2.00 & 0.0968 & $0.1389(0.0059)$ & $0.1265(0.0079)$ & $0.1376(0.0077)$ & $0.1164(0.0119)$ \\
\hline 25 & 0.75 & 1.2162 & $1.4690(0.4384)$ & $1.2518(0.3236)$ & $1.4390(0.3296)$ & $1.2601(0.2432)$ \\
& 1.00 & 0.5149 & $0.6596(0.1127)$ & $0.5683(0.0793)$ & $0.6479(0.0926)$ & $0.5736(0.0712)$ \\
& 1.50 & 0.1850 & $0.2290(0.0146)$ & $0.2022(0.0104)$ & $0.2292(0.0107)$ & $0.2031(0.0085)$ \\
& 2.00 & 0.0968 & $0.1198(0.0033)$ & $0.1091(0.0031)$ & $0.1194(0.0043)$ & $0.1047(0.0034)$ \\
\hline 50 & 0.75 & 1.2162 & $1.3714(0.2381)$ & $1.2376(0.0796)$ & $1.2969(0.1199)$ & $1.2159(0.1036)$ \\
& 1.00 & 0.5149 & $0.5773(0.0410)$ & $0.5402(0.0328)$ & $0.5837(0.0414)$ & $0.5442(0.0269)$ \\
& 1.50 & 0.1850 & $0.2037(0.0048)$ & $0.1920(0.0042)$ & $0.2057(0.0036)$ & $0.1937(0.0032)$ \\
& 2.00 & 0.0968 & $0.1061(0.0013)$ & $0.1000(0.0012)$ & $0.1059(0.0014)$ & $0.0992(0.0013)$ \\
\hline
\end{tabular}

For a given $n$, against each $\alpha$, the first figure represents the average value of the estimate and the figure inside the parentheses is the corresponding variance.

slightly better performance.

\section{Illustrative EXAmPle}

In this section, we illustrate the inferential results in preceding sections by analyzing a data set originally due to Bjerkedal [6], which has also been analyzed previously by Gupta et al. [13]. The data represent the survival times of guinea pigs injected with different doses of tubercle bacilli. It is known that guinea pigs have high susceptibility to human tuberculosis and that is why they were used in this study. Here, we are primarily concerned with the animals in the same cage that were under the same regimen. The regimen number is the common logarithm of the number of bacillary units in $0.5 \mathrm{ml}$. of challenge solution; i.e., 


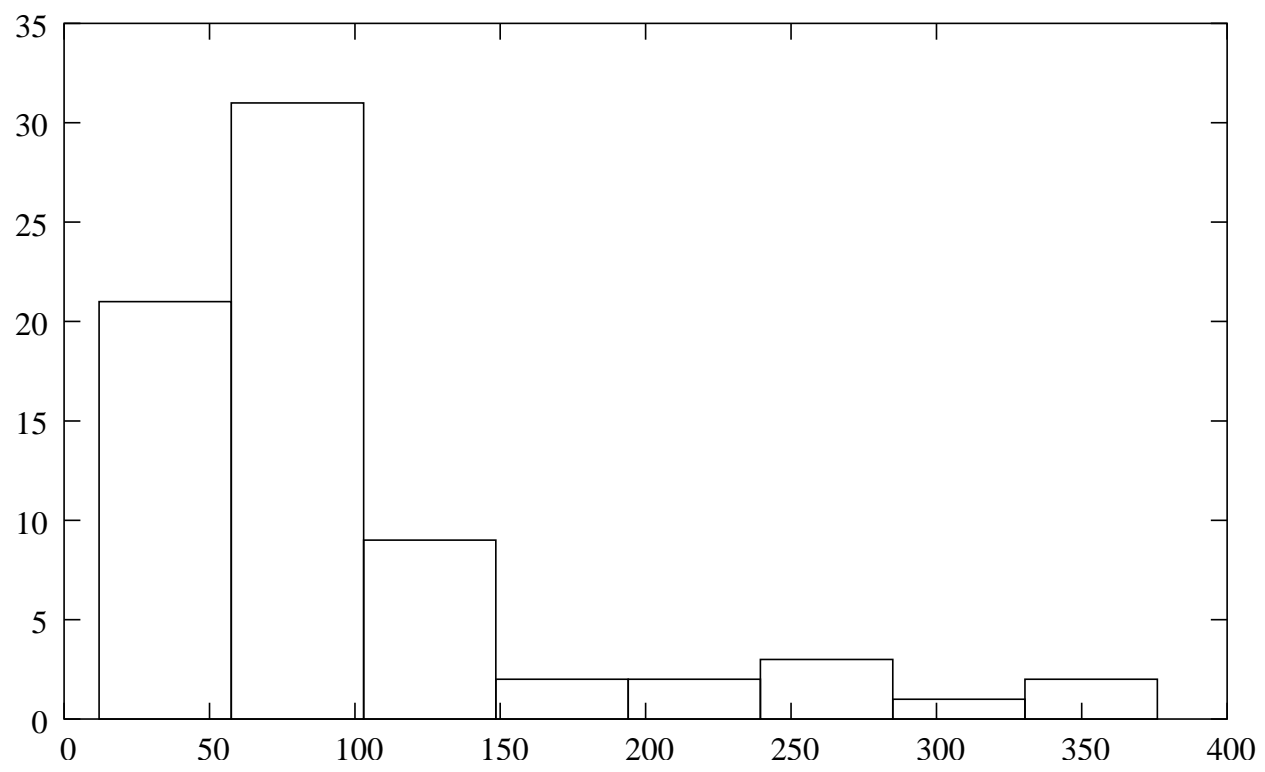

Figure 3: Histogram of the survival times of guinea pigs in Regimen 6.6 data

regimen 6.6 corresponds to $4.0 \times 10^{6}$ bacillary units per $0.5 \mathrm{ml}$. $\left(\log \left(4.0 \times 10^{6}\right)=6.6\right)$.

Corresponding to regimen 6.6, there were 72 observations listed below:

$12,15,22,24,24,32,32,33,34,38,38,43,44,48,52,53,54,54,55,56,57,58,58,59,60$, $60,60,60,61,62,63,65,65,67,68,70,70,72,73,75,76,76,81,83,84,85,87,91,95,96$, $98,99,109,110,121,127,129,131,143,146,146,175,175,211,233,258,258,263,297$, $341,341,376$.

The mean, standard deviation and the coefficient of skewness are calculated as 99.82, 80.55 and 1.80, respectively. The skewness measure indicates that the data are positively skewed. The histogram plot, presented in Figure 3, also supports this point. Moreover, Gupta et al. [13] observed that the empirical hazard function calculated from these data is unimodal. We, therefore, use the BS distribution to analyze these data. We determined the MMEs of $\alpha$ and $\beta$ as 0.7707 and 77.2931, respectively, and the BCMMEs as 0.7600 and 77.4525. The plots of empirical survival function and the fitted survival function are presented in Figure 4. They are nearly identical. Furthermore, the Kolmogorov-Smirnov (KS) distance between 


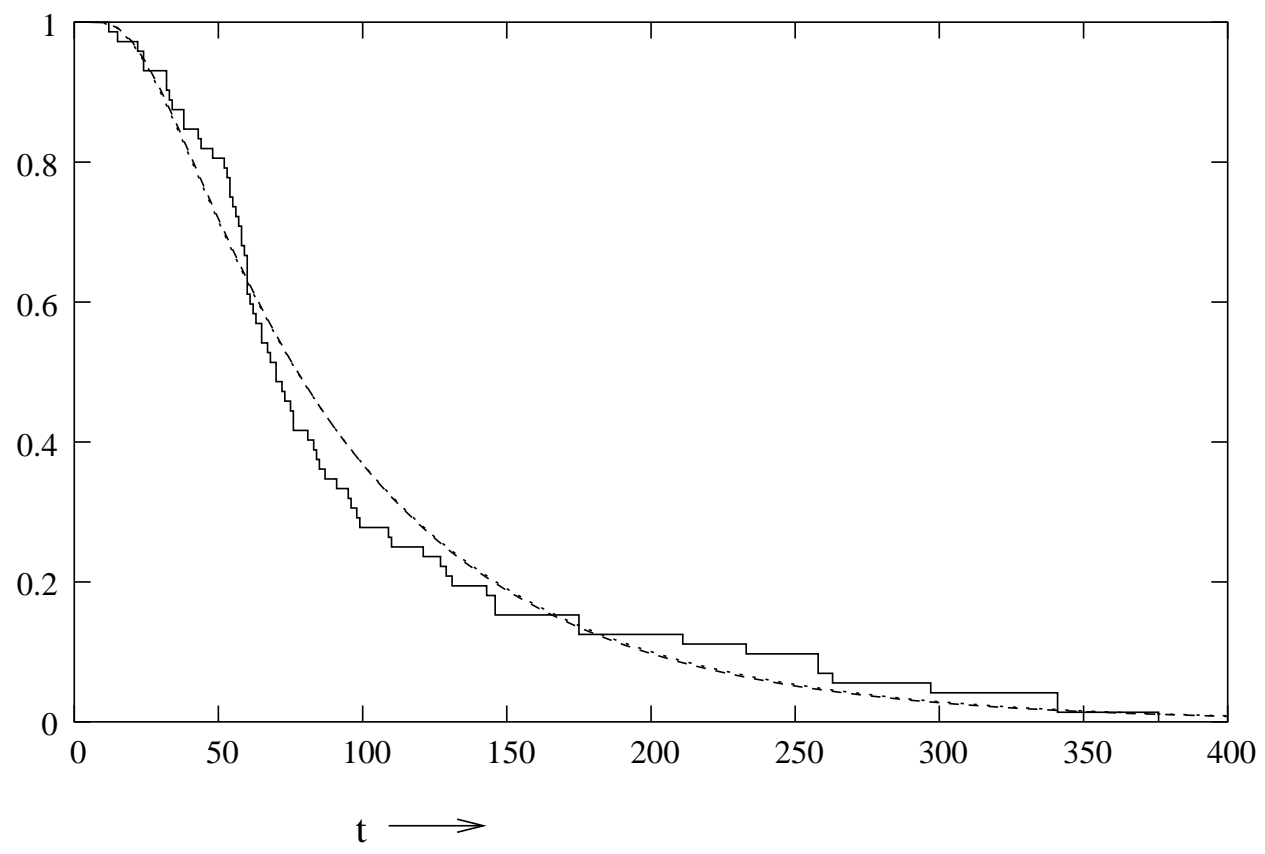

Figure 4: The empirical survival function and the fitted survival functions of the guinea pigs in Regimen 6.6 data

the empirical and fitted survival functions is found to be 0.1044 with the corresponding $p$ value being 0.4125 , which clearly suggests that the BS distribution provides a good fit to the Guinea pig data. The plots of the estimated hazard functions based on the MMEs and BCMMEs are presented in Figure 5. We also computed the MME and the AMME of the change point $c_{\alpha, \beta}$ to be 90.30 and 87.80 , respectively, and the corresponding $95 \%$ bootstrap confidence intervals to be $(59.37,137.55)$ and $(59.31,131.44)$. Similarly, we computed the BCMME and ABCMME to be 86.20 and 84.05, respectively, and the corresponding $95 \%$ bootstrap confidence intervals to be $(57.84,134.96)$ and $(57.92,128.61)$. As mentioned earlier, we observe the results obtained by the two methods to be quite close. 


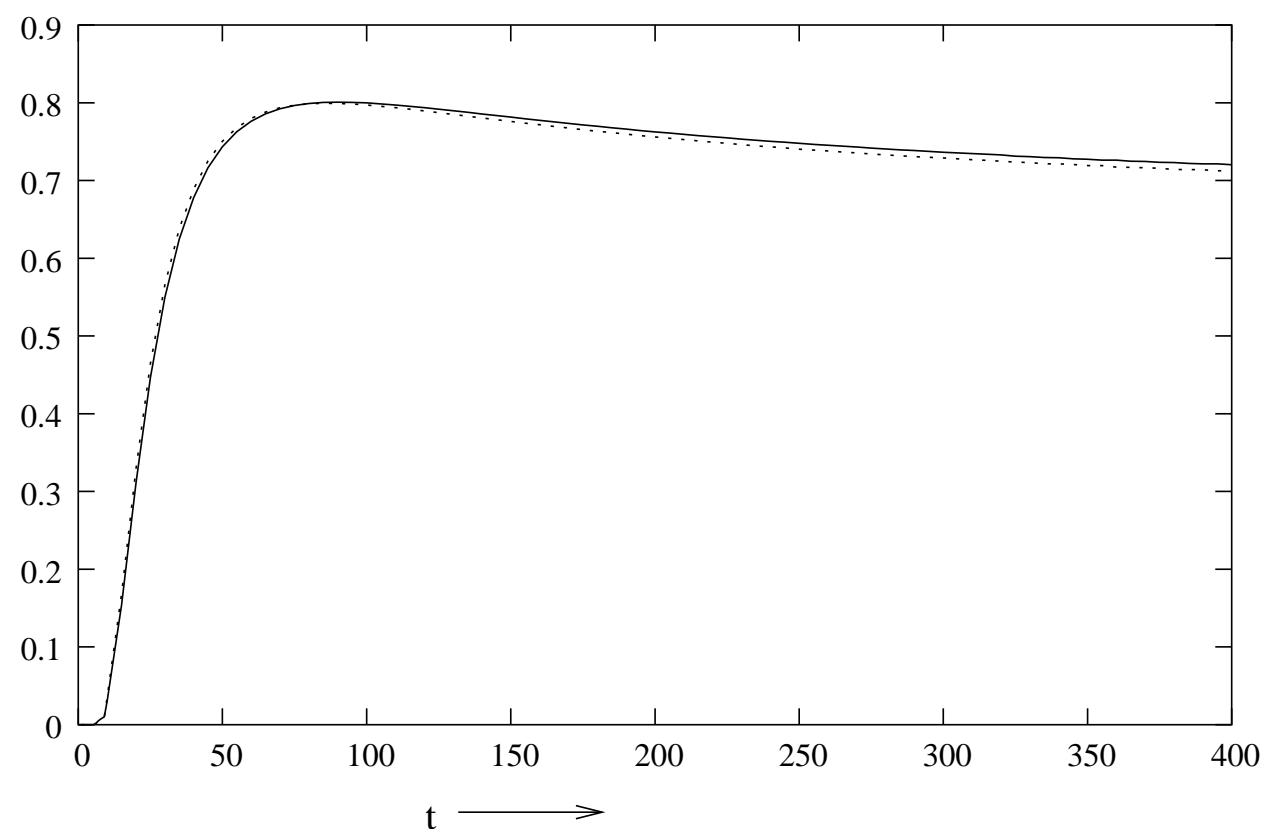

Figure 5: The estimated hazard functions based on MMEs and BCMMEs

\section{Conclusions}

In this paper, we have shown that the hazard function of the two-parameter BS distribution is unimodal (of upside down form). The change point in the hazard function can be obtained as a solution of a non-linear equation. We have provided an approximation to this change point and have shown that the approximation works very well whenever the shape parameter is not too small. We have proposed different methods for estimating the change point and have compared their performance through Monte Carlo simulations. We have demonstrated that both BCMME and ABCMME perform well, and since ABCMME is a simple explicit estimator, we have recommended its usage for the estimation of change point. We have derived the asymptotic distribution of all the estimators and because of their complexity, we have used bootstrap confidence intervals for data analysis. 


\section{APPENDIX}

Here, we derive the asymptotic distributions of the estimates $c_{\alpha, \beta}$ by $c_{\hat{\alpha}, \hat{\beta}}$ and $c_{\tilde{\alpha}, \tilde{\beta}}$, which can be used to construct confidence intervals for the unknown change point $c_{\alpha, \beta}$. $\mathrm{Ng}$ et al. [18] showed that the asymptotic joint distribution of $\hat{\alpha}$ and $\hat{\beta}$ is bivariate normal

$$
\left(\begin{array}{c}
\hat{\alpha} \\
\hat{\beta}
\end{array}\right) \sim N\left[\left(\begin{array}{c}
\alpha \\
\beta
\end{array}\right),\left(\begin{array}{cc}
\frac{\alpha^{2}}{2 n} & 0 \\
0 & \frac{(\alpha \beta)^{2}}{n}\left(\frac{1+\frac{3}{4} \alpha^{2}}{\left(1+\frac{1}{2} \alpha^{2}\right)^{2}}\right)
\end{array}\right)\right] .
$$

From this distributional result, the asymptotic distribution of $c_{\hat{\alpha}, \hat{\beta}}$ can be obtained by employing the standard delta-method. It can be shown that it is univariate normal

$$
c_{\hat{\alpha}, \hat{\beta}} \sim N\left(c_{\alpha, \beta}, \frac{A}{n}\right),
$$

where

$$
A=\frac{\alpha^{2}}{2}\left(\frac{\frac{\partial^{2} h(t, \alpha, \beta)}{\partial t \partial \alpha}}{\frac{\partial^{2} h(t, \alpha, \beta)}{\partial t \partial t}}\right)^{2}+(\alpha \beta)^{2}\left(\frac{1+\frac{3}{4} \alpha^{2}}{\left(1+\frac{1}{2} \alpha^{2}\right)^{2}}\right)\left(\frac{\frac{\partial^{2} h(t, \alpha, \beta)}{\partial t \partial \beta}}{\frac{\partial^{2} h(t, \alpha, \beta)}{\partial t \partial t}}\right)^{2} .
$$

$\mathrm{Ng}$ et al. [18] also showed that the asymptotic joint distribution of $\tilde{\alpha}$ and $\tilde{\beta}$ is bivariate normal

$$
\left(\begin{array}{c}
\tilde{\alpha} \\
\tilde{\beta}
\end{array}\right) \sim N\left[\left(\begin{array}{c}
\alpha \\
\beta
\end{array}\right),\left(\begin{array}{cc}
\frac{n \alpha^{2}}{2(n-1)^{2}} & 0 \\
0 & \frac{16 n(\alpha \beta)^{2}}{\left(4 n+\alpha^{2}\right)^{2}}\left(\frac{1+\frac{3}{4} \alpha^{2}}{\left(1+\frac{1}{2} \alpha^{2}\right)^{2}}\right)
\end{array}\right)\right]
$$

using which the asymptotic distribution of $c_{\tilde{\alpha}, \tilde{\beta}}$ can be shown to be univariate normal as follows:

$$
c_{\tilde{\alpha}, \tilde{\beta}} \sim N\left(c_{\alpha, \beta}, \frac{B}{n}\right)
$$

where

$$
B=\frac{\alpha^{2} n^{2}}{2(n-1)^{2}}\left(\frac{\frac{\partial^{2} h(t, \alpha, \beta)}{\partial t \partial \alpha}}{\frac{\partial^{2} h(t, \alpha, \beta)}{\partial t \partial t}}\right)^{2}+\frac{(\alpha \beta)^{2}}{\left(1+\frac{\alpha^{2}}{4 n}\right)^{2}}\left(\frac{1+\frac{3}{4} \alpha^{2}}{\left(1+\frac{1}{2} \alpha^{2}\right)^{2}}\right)\left(\frac{\frac{\partial^{2} h(t, \alpha, \beta)}{\partial t \partial \beta}}{\frac{\partial^{2} h(t, \alpha, \beta)}{\partial t \partial t}}\right)^{2} .
$$




\section{ACKNOWLEDGEMENTS}

The authors would like to thank two referees and the associate editor for their constructive comments and suggestions which led to a considerable improved on an earlier version of this paper.

\section{References}

[1] Badar, M. G. and Priest, A. M. (1982). "Statistical aspects of fiber and bundle strength in hybrid composites", Progress in Science and Engineering Composites, Hayashi, T., Kawata, K. and Umekawa, S. (Eds.), ICCM-IV, Tokyo, 1129-1136.

[2] Balakrishnan, N., Leiva, V., Lopez, J. (2007), "Acceptance sampling plans from truncated life tests based on the generalized Birnbaum-Saunders distribution", Communications in Statistics - Simulation and Computation, vol. 36, 643-656.

[3] Bennett, S. (1983). "Logistic regression model for survival data", Applied Statistics, vol. $32,165-171$.

[4] Birnbaum, Z. W. and Saunders, S. C. (1969). "A new family of life distribution", Journal of Applied Probability, vol. 6, 319-327.

[5] Birnbaum, Z. W. and Saunders, S. C. (1969). "Estimation for a family of life distributions with applications to fatigue", Journal of Applied Probability, vol. 6, 328-347.

[6] Bjerkedal, T. (1960). "Acquisition of resistance in guinea pigs infected with different doses of virulent tubercle bacilli", American Journal of Hygiene, vol. 72, 130-148.

[7] Chang, D. S. and Tang, L. C. (1993). "Reliability bounds and critical time for the Birnbaum-Saunders distribution", IEEE Transactions on Reliability, vol. 42, 464-469. 
[8] Chang, D. S. and Tang, L. C. (1994). "Percentile bounds and tolerance limits for the Birnbaum-Saunders distribution", Communications in Statistics - Theory and Methods, vol. 23, 2853-2863.

[9] Desmond, A. F. (1985). "Stochastic models of failure in random environments", Canadian Journal of Statistics, vol. 13, 171-183.

[10] Dupuis, D. J. and Mills, J. E. (1998). "Robust estimation of the Birnbaum-Saunders distribution", IEEE Transactions on Reliability, vol. 47, 88-95.

[11] From, S.G., Li, L.X. (2006). "Estimation of the parameters of the Birnbaum-Saunders distribution", Communications in Statistics - Theory and Methods, vol. 35, 2157-2169.

[12] Glaser, R. A. (1980). "Bathtub and related failure rate characterization", Journal of the American Statistical Association, vol. 75, 667-672.

[13] Gupta, R. C., Kannan, N. and RayChoudhuri, A. (1997). "Analysis of log-normal survival data", Mathematical Biosciences, vol. 139, 103-115.

[14] Johnson, N. L., Kotz, S. and Balakrishnan, N. (1995). Continuous Univariate Distributions - Vol. 2, Second edition, John Wiley \& Sons, New York.

[15] Langlands, A. O., Pocock, S. J., Kerr, G. R. and Gore, S. M. (1979). "Long term survival of patients with breast cancer: A study of the curability of the disease", British Medical Journal, vol. 17, 1247-1251.

[16] Lemonte, A.J., Cribari-Neto, F., Vasconcellos, K.L.P. (2007). "Improved statistical inference for the two-parameter Birnbaum-Saunders distribution", Computational Statistics \& Data Analysis, vol. 51, 4656-4681.

[17] Mann, N. R., Schafer, R. E., and Singpurwalla, N. D. (1974). Methods for Statistical Analysis of Reliability and Life Data, John Wiley \& Sons, New York. 
[18] Ng, H. K. T., Kundu, D. and Balakrishnan, N. (2003). "Modified moment estimation for the two-parameter Birnbaum-Saunders distribution", Computational Statistics \& Data Analysis, vol. 43, 283-298.

[19] Ng, H. K. T., Kundu, D. and Balakrishnan, N. (2006). " Point and interval estimation for the two-parameter Birnbaum-Saunders distribution based on Type-II censored samples", Computational Statistics $\&$ Data Analysis, vol 50, 3222-3242.

[20] Owen, W.J. (2006). "A new three-parameter extension to the Birnbaum-Saunders distribution" IEEE Transactions on Reliability, vol. 55, 475-479.

[21] Pinelis, I. (2002). "Monotonicity properties of the relative error of a Pade approximation for Mills' ratio", Journal of Inequalities in Pure and Applied Mathematics (electronic), vol. 3, Article 20.

[22] Press, W. H., Flannery, B. P., Teukolsky, S. A. and Vetterling, W. T. (1991). Numerical Recipes: The Art of Scientific Computing, Cambridge University Press, Cambridge, U.K.

[23] Rieck, J. R. (1995). "Parametric estimation for the Birnbaum-Saunders distribution based on symmetrically censored samples", Communications in Statistics - Theory and Methods, vol. 24, 1721-1736.

[24] Rieck, J. R. (1999). "A Moment-generating function with application to the BirnbaumSaunders distribution", Communications in Statistics - Theory and Methods, vol. 28, 2213-2222.

[25] Sarles, J. G. and Padgett, W. J. (1998). "Inference for reliability and stress-strength for a scaled Burr-type X distribution", Lifetime Data Analysis, vol. 7, 187-200.

[26] Xie, F.C. and Wei, B.C. (2007). "Diagnostics analysis for log-Birnbaum-Saunders regression models", Computational Statistics 6 Data Analysis, vol. 51, 4692-4706. 